\title{
Angioplastia con balón de venas pulmonares por estenosis tras ablación percutánea de fibrilación auricular
}

\author{
Balloon angioplasty of pulmonary vein stenosis after percutaneous ablation of atrial \\ fibrillation \\ Natalia Campo-Rivera ${ }^{1}$, Alberto Negrete-Salcedo², Carlos Tenorio-Tascón², Alicia Rodríguez-Martínez ${ }^{2}$ y \\ William Escobar-Rojas ${ }^{2}$ \\ ${ }^{1}$ Departamento de Medicina Interna, Universidad Libre; ${ }^{2}$ Servicio de Electrofisiología y Marcapasos, Clínica Imbanaco. Cali, Colombia
}

\section{Resumen}

Se presenta el caso de un hombre de 31 años, con historia de fibrilación auricular paroxística, a quien se realizó exitosamente una ablación por radiofrecuencia de venas pulmonares guiada por un sistema de mapeo tridimensional EnSite ${ }^{T M}$. Tres meses después del procedimiento presentó hemoptisis y dolor torácico de características pleuríticas, motivo por el cual se le realizó una angiotomografía computarizada coronaria que evidenció una estenosis grave de la vena pulmonar superior izquierda y una estenosis moderada de la vena inferior izquierda. El paciente fue sometido a angioplastia con balón, con la cual se normalizó la perfusión pulmonar.

Palabras clave: Fibrilación auricular. Ablación por catéter. Estenosis de venas pulmonares.

\section{Abstract}

We report the case of a 31-year-old male patient with a history of paroxysmal atrial fibrillation, who underwent a successful radiofrequency pulmonary vein ablation using EnSite ${ }^{T M}$ three-dimensional mapping system. Three months after the procedure, patient presented with hemoptysis and pleuritic chest pain. A coronary computed tomography angiography was performed, which showed a severe left superior pulmonary vein stenosis and a moderate left inferior pulmonary vein stenosis. A balloon angioplasty was performed with subsequent restoration of pulmonary perfusion.

Key words: Atrial fibrillation. Catheter ablation. Pulmonary vein stenosis.

\section{Introducción}

La fibrilación auricular es una alteración del ritmo cardiaco caracterizada por la contracción rápida (entre 400 y 700 veces por minuto) e irregular de las aurículas. Es la arritmia sostenida más frecuente, con una prevalencia del $0.5 \%$ en la población general'. Se asocia a una gran morbilidad y una alta mortalidad debido a que incrementa sustancialmente el riesgo de desarrollo y deterioro de insuficiencia cardiaca congestiva, y de la aparición de eventos cerebrovasculares como resultado de cardioembolia proveniente de la aurícula izquierda ${ }^{2}$. Debido a esto, en los últimos años se ha incrementado el uso de estrategias invasivas

\section{Correspondencia:}

*Natalia Campo-Rivera

E-mail: natalicamporivera @gmail.com
Disponible en internet: 29-11-2021 Rev Colomb Cardiol. 2021;28(5):473-477 www.rccardiologia.com 0120-5633 / @ 2020 Sociedad Colombiana de Cardiología y Cirugía Cardiovascular. Publicado por Permanyer. Este es un artículo open access bajo la licencia CC BY-NC-ND (http://creativecommons.org/licenses/by-nc-nd/4.0/). 
encaminadas a mantener el ritmo sinusal, como es la realización de ablación de las venas pulmonares por radiofrecuencia. Tras esta, en pacientes seleccionados correctamente, se espera que el $50-75 \%$ permanezcan libres de arritmia en el primer año, respecto al 10-30\% de los tratados con medicamentos antiarrítmicos ${ }^{3}$.

La ablación por radiofrecuencia se considera un procedimiento seguro, pero no exento de complicaciones. Por fortuna, estas suelen ser infrecuentes y autolimitadas; muy ocasionalmente pueden comprometer el estado general del paciente y requerir intervenciones adicionales, como es el caso de las estenosis de las venas pulmonares.

La estenosis grave (reducción $\geq 70 \%$ diámetro) de las venas pulmonares como consecuencia de ablación circunferencial y aislamiento antral tiene una incidencia aproximada del $0.6 \%$. Esta complicación solía ser frecuente cuando se realizaba ablación segmental o focal a nivel de los ostia de las venas pulmonares ${ }^{4}$.

Entre los factores de riesgo identificados para el desarrollo de esta complicación se encuentran la edad avanzada y la presencia de un ostium con gran sección transversal; en el aspecto técnico están la mayor duración, la temperatura y la potencia de aplicación de radiofrecuencia con el catéter irrigado ${ }^{5}$.

En la actualidad no se recomienda realizar tamizaje para estenosis de venas pulmonares. Los estudios de imagen que confirman esta complicación suelen ser realizados en pacientes sintomáticos que consultan meses después del procedimiento. La presentación clínica más frecuente (disnea durante el reposo, disminución de la capacidad para realizar ejercicio, tos) se asemeja a la de otras enfermedades cardiacas y pulmonares, y el hallazgo de consolidación parenquimatosa atribuible a hemorragia venosa puede ser confundido con un proceso neumónico e incluso con malignidad $^{6}$. Por estas razones, generalmente hay un retraso en el diagnóstico, lo que lleva a un incremento del riesgo de progresión de la estenosis, oclusión y fibrosis, y predispone al desarrollo de daño pulmonar grave intraparenquimatoso e infarto pulmonar?

El tratamiento de esta complicación consiste en la realización de angioplastia con balón con o sin implante de stent. A pesar de la instauración de nuevas técnicas percutáneas y del uso de stents medicados, el desarrollo de reestenosis a 3 años es frecuente ${ }^{8}$.

\section{Caso clínico}

Varón de 31 años con antecedente de fibrilación auricular paroxística sintomática de difícil control pese al

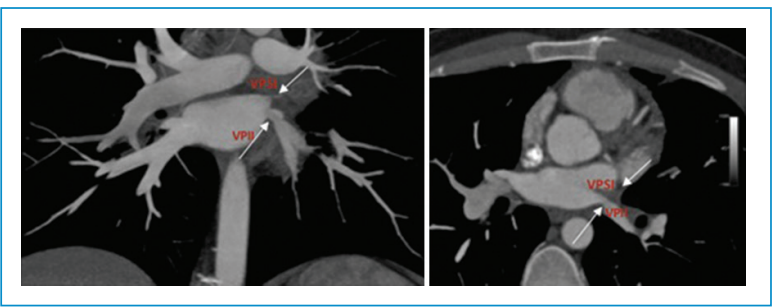

Figura 1. Angiotomografía computarizada coronaria que evidencia la vena pulmonar superior izquierda (VPSI) obliterada y la vena pulmonar inferior izquierda (VPII) con disminución de su calibre.

empleo de múltiples antiarrítmicos, a quien se realizó, con la ayuda de ecografía intracardiaca y mapeo no fluoroscópico EnSite ${ }^{\mathrm{TM}}$, ablación circunferencial y aislamiento antral de venas pulmonares.

Se procedió a localizar el catéter de ablación y el catéter deflectable circular de mapeo (AFocus ${ }^{\mathrm{TM}}$ ) en cada ostium de las venas pulmonares. Adicionalmente, para la comprobación de la posición de los catéteres en la aurícula izquierda se hizo inyección de medio de contraste MD 76. Una vez detectados los potenciales de las venas pulmonares, se procedió a la aplicación de radiofrecuencia con el catéter irrigado. Las aplicaciones se efectuaron con energía entre 20 y $30 \mathrm{~W}$, temperatura máxima de $36.8^{\circ} \mathrm{C}$ y una duración máxima de 120 segundos en cada sitio de aplicación. Se corroboró la desaparición de dichos potenciales o su disminución en voltaje a menos de $0.5 \mathrm{mV}$. El aislamiento eléctrico de los ostia de las venas también se confirmó con mapeo no fluoroscópico EnSite ${ }^{\mathrm{TM}}$.

Tres meses después, el paciente acudió al servicio de urgencias debido a tos, disnea de grandes esfuerzos, esputo sanguinolento y dolor torácico durante la inspiración. Se le realizó una tomografía computarizada de tórax, la cual fue sugestiva de hemorragia alveolar en el lóbulo superior izquierdo y en la língula, junto con derrame pleural de disposición libre. Se le realizó una angiotomografía computarizada coronaria, la cual mostró que la vena pulmonar superior izquierda se encontraba obliterada y que la vena pulmonar inferior izquierda presentaba una disminución de su calibre (Fig. 1). Se revisaron las imágenes del mapeo no fluoroscópico EnSite ${ }^{\mathrm{TM}}$ realizado previamente y se hizo evidente la complicación (Fig. 2).

Se realizó un ecocardiograma transtorácico que mostró las cavidades derechas de tamaño y función normales, TAPSE (tricuspid annular plane systolic 
excursión) de $21 \mathrm{~mm}$, sin signos sugestivos de hipertensión pulmonar.

Por la sintomatología descrita y los hallazgos de los estudios de imagen, se decidió realizar angioplastia de venas pulmonares izquierdas.

A través de la vena femoral derecha se pasó un introductor Mullins y con aguja de Brockenbrough se realizó punción transeptal guiada por ecocardiograma intracardiaco. Se localizaron los ostia de las venas pulmonares y se realizó venografía de las venas pulmonares izquierdas, demostrándose estenosis grave de la vena superior y estenosis moderada de la vena inferior.

Se realizó angioplastia con balón Powerflex ${ }^{\mathrm{TM}}$ de $6 \times$ $20 \mathrm{~mm}$ y $8 \times 20 \mathrm{~mm}$, observándose mejoría significativa de la luz y de los flujos en los vasos (Fig. 3). En vista de la adecuada dilatación de las venas, se decidió no implantar stents. Treinta minutos después se visualizaron las venas pulmonares izquierdas nuevamente $y$ no se observó ningún tipo de retracción.

El paciente presentó resolución del esputo sanguinolento, la tos y la disnea tras el procedimiento, y ha continuado asintomático durante el seguimiento.

\section{Discusión}

La ablación de la fibrilación auricular por radiofrecuencia es una estrategia terapéutica eficaz y segura para el control del ritmo. Sus complicaciones han disminuido en incidencia a partir del uso de sistemas de mapeo electroanatómico tridimensional y de técnicas de ablación circunferencial y aislamiento antral.

La ablación con catéter para el tratamiento de la fibrilación auricular presenta una mortalidad periprocedimiento menor del $0.2 \%$. Las complicaciones potencialmente fatales, pero usualmente manejables, ocurren en un $2-3 \%$ de los pacientes intervenidos, y son el accidente cerebrovascular periprocedimiento, la perforación o fístula esofágica, y el taponamiento cardiaco. Entre las complicaciones graves más frecuentes se encuentran la parálisis persistente del nervio fréni$\mathrm{co}$, las lesiones vasculares y la estenosis de las venas pulmonares ${ }^{8}$.

El riesgo de estenosis de las venas pulmonares, definido como una reducción $\geq 3 \mathrm{~mm}$ de su diámetro, depende primordialmente de las técnicas de ablación empleadas. El riesgo de estenosis grave tras una ablación por radiofrecuencia de las venas pulmonares era mucho mayor cuando se realizaba ablación segmental o focal a nivel de los ostia de las venas pulmonares (ocurría hasta en un $21 \%$ de los pacientes), comparado

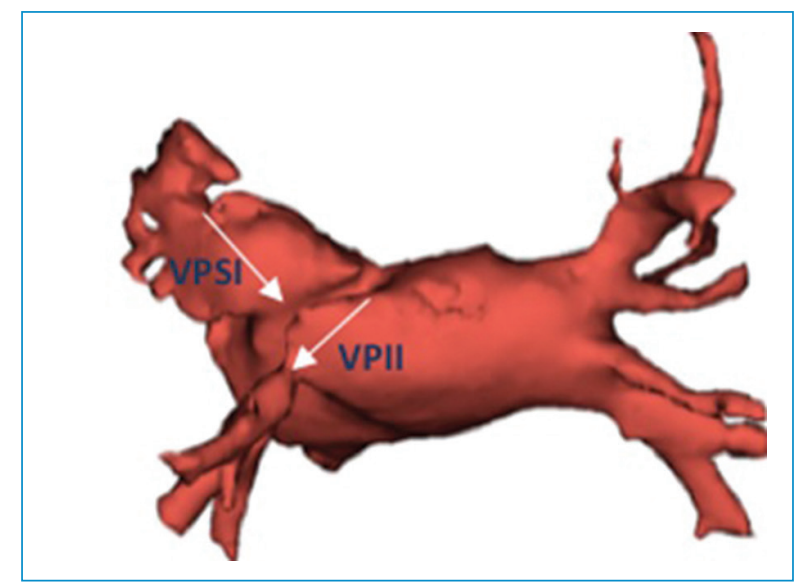

Figura 2. Reconstrucción tridimensional que evidencia una estenosis grave de la vena pulmonar superior izquierda (VPSI) y una estenosis moderada de la vena pulmonar inferior izquierda (VPII).

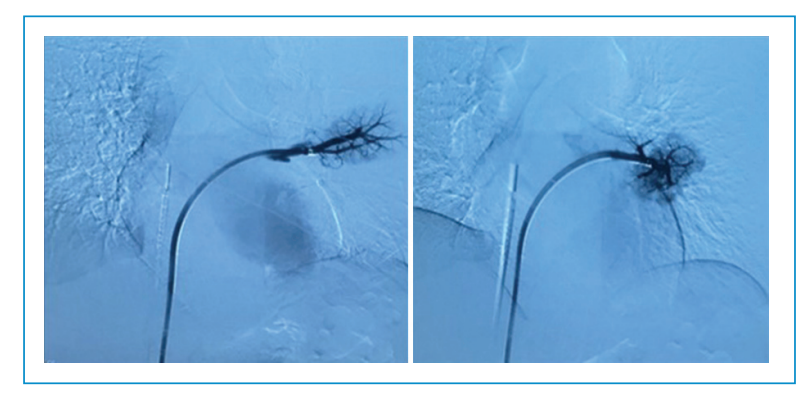

Figura 3. Se corrobora la recuperación del flujo en la vena pulmonar superior izquierda y la vena pulmonar inferior izquierda tras la angioplastia con balón.

con el riesgo asociado al empleo de la técnica actual de ablación circunferencial y aislamiento antral, con la cual se estima que tan solo el $0.6 \%$ de los pacientes desarrollan una estenosis grave ${ }^{4}$.

Además de la ablación por radiofrecuencia utilizada en el paciente del caso clínico descrito, para el tratamiento de la fibrilación auricular se puede emplear la crioablación con catéter-balón. Entre sus ventajas destaca el menor tiempo requerido para el aprendizaje de la técnica, y entre sus desventajas, que expone tanto al paciente como al médico a un tiempo más largo de fluoroscopia?

Se ha planteado que la crioablación con catéter-balón podría reducir el riesgo de desarrollo de estenosis de las venas pulmonares, pero los hallazgos hasta la fecha no son concluyentes. En el ensayo clínico FIRE AND ICE, en el que se intervino a 750 personas, no hubo diferencias estadísticamente significativas respecto a los desenlaces de seguridad entre ambas técnicas; ninguno 
de los pacientes evaluados en el grupo de ablación con radiofrecuencia ni en el grupo de crioablación con catéter-balón presentó síntomas sugestivos de estenosis de las venas pulmonares durante un seguimiento promedio de un año y medio ${ }^{9}$. Sin embargo, en un estudio retrospectivo, monocéntrico, en el cual se realizó a todos los pacientes una tomografía computarizada de aurícula izquierda y venas pulmonares, se evidenció que 12 (3.1\%) de los intervenidos con ablación por radiofrecuencia desarrollaron estenosis de las venas pulmonares; en contraste, solo un paciente $(0.4 \%)$ de los intervenidos con crioablación con catéter-balón presentó estenosis de las venas pulmonares, y esta diferencia fue estadísticamente significativa ${ }^{10}$. Cabe señalar que cuando se realizan estudios de imagen de manera sistemática se diagnostican pacientes con estenosis leves y moderadas de las venas pulmonares sin repercusión clínica, y de ahí que la prevalencia de esta complicación en este estudio retrospectivo fuera mayor.

Existen dos opciones de tratamiento en caso de estenosis grave y sintomática de las venas pulmonares: la angioplastia con balón y la angioplastia con balón más implante de stent. Cada caso deberá ser individualizado, y las características de la respuesta de las venas a la angioplastia con balón serán determinantes para decidir si es necesario o no implantar un stent ${ }^{11}$.

En un estudio prospectivo, observacional, de 124 pacientes con estenosis grave de las venas pulmonares, se consideró realizar angioplastia con balón de las venas pulmonares sin implante de stent cuando, tras posicionar el balón en el punto de estenosis máxima, se lograba la restauración del flujo, corroborándose la patencia de las venas con inyecciones repetidas de contraste. En caso de persistir una estenosis mayor del $20 \%$, o en caso de retroceso elástico venoso, se realizaba implante de stent. Cuando se implantó un stent, los pacientes recibieron clopidogrel y anticoagulación con warfarina de manera indefinida. Es importante destacar que el riesgo de reestenosis es mayor cuando no se implanta un stent; en este estudio, 23 (27\%) pacientes desarrollaron reestenosis cuando se implantó un stent, frente a $52(57 \%)$ pacientes tratados con angioplastia con balón ${ }^{11}$.

La estenosis grave de las venas pulmonares tras la ablación percutánea de la fibrilación auricular, al ser un desenlace adverso infrecuente y tener manifestaciones clínicas y hallazgos radiológicos en el parénquima pulmonar no característicos, suele ser diagnosticada de manera tardía, hecho que incrementa el riesgo de progresión de la gravedad de la estenosis, la posibilidad de fibrosis de las venas y la gravedad del daño pulmonar asociado ${ }^{12}$.
El paciente del caso descrito presentó, secundario a una ablación circunferencial y aislamiento antral con catéter irrigado guiado por sistema de mapeo no fluoroscópico EnSite ${ }^{T M}$ (Fig. 2), oclusión de la vena pulmonar superior izquierda y estenosis moderada de la vena pulmonar inferior izquierda. La sintomatología (hemoptisis y dolor pleurítico) se inició 3 meses después de la ablación por radiofrecuencia. El diagnóstico fue realizado por angiotomografía computarizada coronaria, y en ese momento el paciente presentaba hemorragia alveolar en el lóbulo superior izquierdo y la língula, sin compromiso estructural cardiaco.

Se decidió solicitar una angiotomografía computarizada coronaria como imagen diagnóstica, dado que los hallazgos en la tomografía computarizada de tórax no permitieron identificar la etiología del cuadro clínico presentado por el paciente; a su vez, ante la posibilidad de estenosis de las venas pulmonares, con este estudio pueden realizarse una reconstrucción tridimensional y una medición precisa del diámetro luminal de las venas pulmonares ${ }^{13}$, y como elemento adicional permite analizar la anatomía coronaria con gran exactitud ${ }^{14}$.

En el caso descrito se consideró la angioplastia con balón sin colocación de stent, debido a que se evidenció una recuperación completa del flujo de manera inmediata y recuperación total de los diámetros de ambas venas pulmonares izquierdas tras la angioplastia; se observó patencia de los vasos tras el procedimiento, lo cual se corroboró mediante fluoroscopia realizada 30 minutos después de la angioplastia con balón; se consideró que el implante de un stent en esta posición no garantiza la permeabilidad de las venas a largo plazo; se tuvo presente que la posible reestenosis del stent podría repercutir enormemente en el estado de salud del paciente; y se evitó tener que poner tratamiento antiagregante y anticoagulante a un paciente con fibrilación auricular con $\mathrm{CHADS}_{2}$-VASc de 0 , dado que las condiciones sociodemográficas del paciente permiten un seguimiento clínico e imagenológico estricto.

\section{Conclusiones}

La estenosis de las venas pulmonares es una complicación infrecuente de la ablación percutánea de la fibrilación auricular. Es una condición de difícil diagnóstico debido a que la sintomatología característica es inespecífica. Por el momento, existe debate en cuanto a las opciones terapéuticas y la decisión deberá ser siempre individualizada. En este reporte de caso se ilustra cómo la angioplastia con balón es una estrategia segura y 
eficaz para retornar el flujo en estos vasos, lo cual se refleja, a su vez, en una mejoría clínica del paciente.

\section{Financiamiento}

Los autores declaran no haber recibido financiamiento.

\section{Conflicto de intereses}

Los autores declaran que no tienen ningún conflicto de intereses.

\section{Responsabilidades éticas}

Protección de personas y animales. Los autores declaran que para esta investigación no se han realizado experimentos en seres humanos ni en animales.

Confidencialidad de los datos. Los autores declaran que han seguido los protocolos de su centro de trabajo sobre la publicación de datos de pacientes.

Derecho a la privacidad y consentimiento informado. Los autores han obtenido el consentimiento informado de los pacientes $y / 0$ sujetos referidos en el artículo. Este documento obra en poder del autor de correspondencia.

\section{Bibliografía}

1. Kannel WB, Abbott RD, Savage DD. Coronary heart disease and atrial fibrillation: the Framingham Study. Am Heart J. 1983;106:389-96.

2. Wang TJ, Larson MG, Levy D. Temporal relations of atrial fibrillation and congestive heart failure and their joint influence on mortality: the Framingham Heart Study. Circulation. 2003;107:2920-5.

3. Jaïs P, Cauchemez B, Macle L, Daoud E, Khairy P, Subbiah R, et al. Catheter ablation versus antiarrhythmic drugs for atrial fibrillation: the A4 study. Circulation. 2008;118:2498-505.

4. Dong J, Vasamreddy CR, Jayam V. Incidence and predictors of pulmonary vein stenosis following catheter ablation of atrial fibrillation using the anatomic pulmonary vein ablation approach: results from paired magnetic resonance imaging. J Cardiovasc Electrophysiol. 2005;16:845-52.

5. Martín-Garre S, Pérez-Castellano N, Quintanilla JG. Predictores de pérdida luminal de venas pulmonares tras ablación por radiofrecuencia. Rev Esp Cardiol. 2015;68:1085-91.

6. Saad EB, Rossillo A, Saad CP. Pulmonary vein stenosis after radiofrequency ablation of atrial fibrillation: functional characterization, evolution, and influence of the ablation strategy. Circulation. 2003;108:3102-7.

7. Tamborero D, Mont L, Nava S. Incidence of pulmonary vein stenosis in patients submitted to atrial fibrillation ablation: a comparison of the selective segmental ostial ablation vs. the circumferential pulmonary veins ablation. J Interv Card Electrophysiol. 2005;14:21-5.

8. Kirchhof P, Benussi S. 2016 ESC Guidelines for the management of atrial fibrillation developed in collaboration with EACTS. Eur Heart J. 2016;37:2893-962.

9. Kuck KH, Brugada J, Fürnkranz A. Cryoballoon or radiofrequency ablation for paroxysmal atrial fibrillation. New Engl J Med. 2016;374:2235-45.

10. Larnier L, Badenco N. Comparison of incidences of pulmonary vein stenosis between radiofrequency and cryoablation in atrial fibrillation ablation. Arch Cardiovasc Dis Suppl. 2018;10:89-90.

11. Fender EA, Widmer RJ. Severe pulmonary vein stenosis resulting from ablation for atrial fibrillation clinical perspective. Circulation. 2016;134: 1812-21.

12. Erin A, Fender R, Widmer J. Severe pulmonary vein stenosis resulting from ablation for atrial fibrillation: presentation, management, and clinical outcomes. Circulation. 2016;134:1812-21.

13. Fender EA, Packer DL, Holmes DR Jr. Pulmonary vein stenosis after atrial fibrillation ablation. Eurolntervention. 2016;12 (Suppl X):X31-4.

14. Bittencourt MS, Blankstein R. Coronary computed tomography angiography: how should we act on what we find? J Nucl Cardiol. 2016;24:1279-81. 